\title{
Multiple Schrödinger Pictures and Dynamics in Shortcuts to Adiabaticity
}

\author{
S. Ibáñez, ${ }^{1}$ Xi Chen, ${ }^{1,2}$ E. Torrontegui, ${ }^{1}$ J. G. Muga, ${ }^{1,2}$ and A. Ruschhaupt ${ }^{3}$ \\ ${ }^{1}$ Departamento de Química-Física, UPV-EHU, Apartado 644, 48080 Bilbao, Spain \\ ${ }^{2}$ Department of Physics, Shanghai University, 200444 Shanghai, People's Republic of China \\ ${ }^{3}$ Department of Physics, University College Cork, Cork, Ireland
}

(Received 22 December 2011; published 7 September 2012)

\begin{abstract}
A Schrödinger equation may be unitarily transformed into dynamical equations in different interaction pictures which describe a common physical process, i.e., the same underlying interactions and dynamics. In contrast to this standard scenario, other relations are also possible, such as a common interactionpicture dynamical equation corresponding to several Schrödinger equations that represent different physical processes. This may enable us to design alternative and feasible experimental routes for operations that are a priori difficult or impossible to perform. The power of this concept is exemplified by engineering Hamiltonians that improve the performance or make realizable several shortcuts to adiabaticity.
\end{abstract}

DOI: 10.1103/PhysRevLett.109.100403

PACS numbers: 03.65.Ca, 03.65.Vf, 32.80.Qk, 42.50.Dv

Introduction.-In standard applications Schrödinger, interaction, or Heisenberg "representations" or "pictures" of a quantum system, are linked to each other by unitary transformations that guarantee their formal equivalence. Changing the picture may be viewed as a change of basis, so in principle the same information can be extracted from any of them. The Schrödinger picture (SP) is often privileged as the primary description, representative of the physical or experimental setting, whereas the multiple interaction pictures (IPs) have the connotation of auxiliary mathematical constructs to facilitate the calculations. The standard relation among them is schematically depicted in Fig. 1(a), where each node may represent the dynamical equations (DEs) for the state vectors, the Hamiltonians, or the state vectors themselves. The external box means that they all represent the same common underlying physics: the same interactions and external forces, and the same system dynamics.

In sharp contrast to this standard application of the interaction picture just described, we propose in this Letter alternative relations such as the ones in Figs. 1(b) and $1(\mathrm{c})$ or more complex combinations, where the nodes may belong to different physical processes, as a way to design alternative and feasible experimental routes for operations that are a priori difficult or impossible to perform. We will thus engineer Hamiltonians which improve or make feasible "shortcuts to adiabaticity" [1]. Adiabatic processes are very common and useful in laboratories, but their intrinsic slowness imposes limitations. The design of alternative fast routes is an active research field of interest in cold atom physics, nuclear magnetic resonance, quantum information processing, and beyond the quantum domain, e.g., to couple different devices in optical communications [2]. Contrary to [1] which is devoted specifically to harmonic oscillator expansions, the schemes proposed here are more general and provide a unified treatment for internal and external degrees of freedom, complementary to existing approaches.

Our first benchmark problem is the acceleration of adiabatic population inversion in a two-level system, in itself a phenomenon of broad interest from NMR applications [3] to quantum information [4]. We shall point out several techniques to eliminate undesired Hamiltonian terms, possible improvements to reinterpreted experiments, and also feasible alternatives to inversion schemes that required cumbersome level-shift engineering or multiple fields [5]. We shall finally show how to overcome the difficulties to implement "counterdiabatic" terms and perform fast trap expansions and transport of cold atoms without final excitation [6].

Multiple Schrödinger pictures.-To explain the multiframe schemes of Figs. 1(b) and 1(c) we shall review first some basic equations and notation. SP and IP states are related by a unitary transformation, $\left|\psi_{I}\right\rangle=U^{\dagger}\left|\psi_{S}\right\rangle,\left|\psi_{S}\right\rangle=$ $U\left|\psi_{I}\right\rangle$, and evolve according to $i \hbar \partial_{t}\left|\psi_{S}\right\rangle=H\left|\psi_{S}\right\rangle$, $i \hbar \partial_{t}\left|\psi_{I}\right\rangle=H_{I}\left|\psi_{I}\right\rangle$, where $H$ is the Hamiltonian in the SP and $H_{I}=U^{\dagger}(H-K) U$, with $K=i \hbar \dot{U} U^{\dagger}$, the corresponding IP Hamiltonian. $H, H_{I}$, and $K$ may be generally time dependent. Note that when $U(t)=1$ at initial and final times the states are equal in both pictures at those times. If, in addition, $\dot{U}=0$ at boundary times, the Hamiltonians coincide there also. Similar relations hold for a unitary operator $U^{\prime}$ which defines an interaction picture $I^{\prime}$.

As in Fig. 1(b), an interaction picture DE may be related unitarily to two or more Schrödinger equations with Hamiltonians that represent different experimental settings and external interactions; i.e., in this case $S$ and $S^{\prime}$ correspond to different physical realities. There is no contradiction with the equivalence discussed above when we pay attention, not only to the DEs but to the observables as well. A given picture is fully characterized by both the DE and the operators for the observables. Thus Fig. 1(b) admits 
(a)

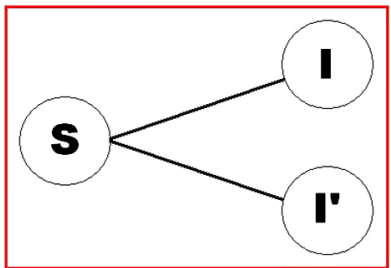

(b)

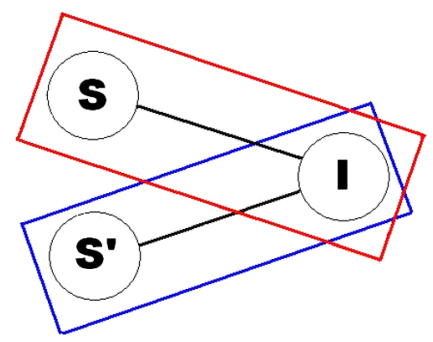

(c)

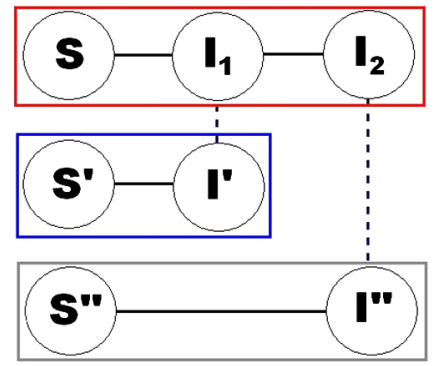

FIG. 1 (color online). Schematic relation between different Schrödinger and interaction picture dynamical equations. Each node may also represent the Hamiltonians or the states. The rectangular boxes enclose nodes that represent the same underlying processes. The solid lines connecting the nodes are unitary relations for the linked states, and the dashed lines represent a nonunitary addition of a term to the Hamiltonian.

several interpretations depending on the treatment given to the observables: If the observables are transformed, from $B_{S}$ in $\mathrm{S}$ to $B_{S^{\prime}}=U^{\prime} U^{\dagger} B_{S} U U^{\prime \dagger}$ in $S^{\prime}$, we will get the same expectation values from two different systems and dynamics performing in general rather different measurement operations. If instead one runs the same measurements in $S$ and $S^{\prime}$ on the same, untransformed observables $B_{S}$, the expectation values will differ in general, and their IP representatives would be either $U^{\dagger} B_{S} U$ or $U^{\prime \dagger} B_{S} U^{\prime}$, sharing in any case a common IP-state dynamics.

Another scheme is depicted in Fig. 1(c). In the upper box a Schrödinger node is related unitarily to a first IP node, $I_{1}$, linked also unitarily to a second one, $I_{2}$. The two consecutive IP Hamiltonians may be modified or perturbed, e.g., by the addition of some terms (dashed lines). Thus the nodes $I^{\prime}$ and $I^{\prime \prime}$ represent different physical processes (middle and lower horizontal boxes) with corresponding Schrödinger dynamics.

Superadiabatic iterations and counterdiabatic corrections.-In the following example the consecutive IPs of Fig. 1(c) are generated by means of adiabatic and superadiabatic iterations $[7,8]$, and the addition of a "counterdiabatic" term in the Hamiltonian is performed so as to avoid transitions, canceling out the $K$ [9]. This enables us to accelerate slow processes without inducing any final excitation. Our starting model Hamiltonian is

$$
H_{j}(t)=\left(\begin{array}{cc}
Z_{j}(t) & X_{j}(t)-i Y_{j}(t) \\
X_{j}(t)+i Y_{j}(t) & -Z_{j}(t)
\end{array}\right),
$$

i.e., $H_{j}=X_{j} \sigma_{x}+Y_{j} \sigma_{y}+Z_{j} \sigma_{z}$ in terms of Pauli matrices. (The index $j$ will be used later to define a series of IP Hamiltonians in successive iterations). The Hamiltonian matrices are expressed in the "bare basis" of the two-level system, $|1\rangle=\left(\begin{array}{l}1 \\ 0\end{array}\right),|2\rangle=\left(\begin{array}{l}0 \\ 1\end{array}\right)$. It could represent several physical systems such as a spin in a magnetic field, a twolevel atom (see below), or a condensate in the bands of an accelerated optical lattice [10,11]. In the later case, $X_{j}$ may be controlled by the trap depth, $Z_{j}$ by the lattice acceleration [10], and a $Y_{j}$ component could in principle be implemented by a second shifted lattice [11]. The Hamiltonian evolution or "trajectory" is specified here by the Cartesian coordinates $X_{j}, Y_{j}, Z_{j}$. Later we shall also use the corresponding polar, azimuthal, and radial spherical coordinates, $\Theta_{j}, \Phi_{j}$, and $R_{j}$.

Let $j=0$ and $Y_{0}=0$. An adiabatic population inversion is achieved with $H_{0}$ by varying slowly $X_{0}$ and $Z_{0}$ so that the resonance is crossed at $Z_{0}=0$, and the eigenvectors of $H_{0}$ interchange their character. Different schemes, such as Landau-Zener, Allen-Eberly [12], and others, may be followed to specify the time dependences.

The first IP that we shall consider depends on the adiabatic basis $\left\{\left|n_{0}(t)\right\rangle\right\}$ that diagonalizes $H_{0}(t)$. Specifically we use $U=A_{0}(t)=\sum_{n=1,2}\left|n_{0}(t)\right\rangle\left\langle n_{0}(0)\right|$, where we assume that the $\left|n_{0}(0)\right\rangle$ coincide with the bare basis. Thus $\left|\psi_{I_{1}}\right\rangle=$ $A_{0}^{\dagger}\left|\psi_{S}\right\rangle$ and $H_{1}=A_{0}^{\dagger}\left(H_{0}-K_{0}\right) A_{0}$, where $K_{0}=i \hbar \dot{A}_{0} A_{0}^{\dagger}$. The phases are chosen so that $\left\langle n_{0}(t) \mid \dot{n}_{0}(t)\right\rangle=0$. This makes $K_{0}$ nondiagonal and minimizes its norm [9,13]. In terms of the polar angle $\Theta_{0}, K_{0}=\hbar\left(\dot{\Theta}_{0} / 2\right) \sigma_{y}$. The adiabatic approximation neglects $K_{0}$ in the IP Hamiltonian $H_{1}$ to trivially solve

$$
i \hbar \partial_{t}\left|\psi_{I_{1}}\right\rangle=A_{0}^{\dagger} H_{0} A_{0}\left|\psi_{I_{1}}\right\rangle,
$$

an uncoupled system in the bare basis. Alternatively one may add $A_{0}^{\dagger} K_{0} A_{0}$ to $H_{1}[5,9,14-16]$. The effect is to cancel any coupling so that Eq. (2) becomes exact rather than an approximation. In the corresponding SP $S^{\prime}$, see the middle box in Fig. 1(c), this amounts to adding the counterdiabatic term $H_{c d}^{(0)}:=K_{0}$ to $H_{0} . H_{0}+K_{0}$ preserves the populations of the approximate adiabatic dynamics even for short process times.

In a new iteration, and similarly for higher orders, we write $H_{1}=A_{0}^{\dagger}\left(H_{0}-K_{0}\right) A_{0}$ in the form of Eq. (1), $j=1$, and diagonalize it to produce a "superadiabatic" basis $\left\{\left|n_{1}(t)\right\rangle\right\}$, and the transformation $A_{1}=\sum_{n=1,2}\left|n_{1}(t)\right\rangle \times$ $\left\langle n_{1}(0)\right|$. As before we assume that this basis coincides at 
the boundary times with the bare basis and that $K_{1}=$ $i \hbar \dot{A}_{1} A_{1}^{\dagger}$ is nondiagonal in $\left\{\left|n_{1}(t)\right\rangle\right\} . A_{1}$ produces a new IP [ $I_{2}$ in Fig. 1(c)] with $\left|\psi_{I_{2}}\right\rangle=A_{1}^{\dagger}\left|\psi_{I_{1}}\right\rangle$, and Hamiltonian $H_{2}=A_{1}^{\dagger}\left(H_{1}-K_{1}\right) A_{1}$. $K_{1}$ can be either neglected to produce a superadiabatic approximation, or canceled by adding a counterdiabatic term. In the corresponding SP [ $S^{\prime \prime}$ in Fig. 1(c)] the Hamiltonian becomes $H_{0}+H_{c d}^{(1)}$, where $H_{c d}^{(1)}=A_{0} K_{1} A_{0}^{\dagger}$ [9]. In that manner a different shortcut Hamiltonian is created. For our reference Hamiltonian $H_{0}$ with $Y_{0}=0$, and using polar angles for $H_{0}$ and $H_{1}$, $H_{c d}^{(1)}=\hbar\left(\dot{\Theta}_{1} / 2\right)\left(\cos \Theta_{0} \sigma_{x}-\sin \Theta_{0} \sigma_{z}\right)$, if $\dot{\Theta}_{0}<0$. The absence of a $Y \sigma_{y}$ component, like in $H_{0}$ and unlike in $H_{c d}^{(0)}$, is in some applications a practical advantage. For example, in an optical lattice implementation of the two-level system only one optical lattice is required [11]. One more advantage of the superadiabatic shortcut is that $H_{c d}^{(1)}$ is less intense (it has a smaller norm) than $H_{c d}^{(0)}$ [9]. Alternative eliminations of $\sigma_{y}$ are discussed next.

Z-axis rotation.-Starting from the SP dynamical equation with $H_{0}+H_{c d}^{(0)}$ that we write now in the form

$$
H_{0}+H_{c d}^{(0)}=\left(\begin{array}{cc}
Z_{0} & P e^{-i \phi} \\
P e^{i \phi} & -Z_{0}
\end{array}\right)
$$

where $\phi=\arctan \left(\hbar \dot{\Theta}_{0} / 2 X_{0}\right), \quad 0 \leq \phi<2 \pi$, and $P=$ $\left[X_{0}^{2}+\left(\hbar \dot{\Theta}_{0} / 2\right)^{2}\right]^{1 / 2}$, we may apply the transformation [17]

$$
U_{z}=\left(\begin{array}{cc}
e^{-i \phi / 2} & 0 \\
0 & e^{i \phi / 2}
\end{array}\right),
$$

which amounts to a rotation about the $Z$ axis by $\phi$. Notice that because $U_{z}$ is diagonal in the bare basis, the bare-state populations are the same in the SP and the IP at all times. In the corresponding IP, and with $K_{z}=i \hbar \dot{U}_{z} U_{z}^{\dagger}$, the interaction Hamiltonian becomes

$$
U_{z}^{\dagger}\left(H_{0}+H_{c d}^{(0)}-K_{z}\right) U_{z}=\left(\begin{array}{cc}
Z_{0}-\hbar \dot{\phi} / 2 & P \\
P & -Z_{0}+\hbar \dot{\phi} / 2
\end{array}\right),
$$

without $Y \sigma_{y}$ component. It can be realized directly in the laboratory (this goes beyond the formal application in [17]) and we may treat it as well as a SP Hamiltonian linked to the $I^{\prime}$ Hamiltonian $A_{0}^{\dagger} H_{0} A_{0}$, a common IP node for the two SP Hamiltonians in Eqs. (3) and (4), connected via $A_{0}$ and $U_{z}^{\dagger} A_{0}$, respectively. This Hamiltonian trio and the corresponding dynamical equations constitute a neat example of the dual scheme of Fig. 1(b). Equation (4) provides an alternative shortcut path, that guarantees the same bare-state populations as $H_{0}+H_{c d}^{(0)}$, and indeed it has been implemented experimentally for a condensate on an accelerating lattice [11], to avoid the realization of a $\sigma_{y}$ term with a second optical lattice. The transition from Eq. (3) to (4) was justified based on properties specific to the optical lattice setting in [11].
In fact the elimination of $\sigma_{y}$ in the Hamiltonian can be done formally for any physical realization, and its usefulness will depend on the feasibility to implement the modified $X$ and $Z$ terms, demonstrated for a condensate on an accelerating lattice [11], but more involved for a two-level atom in an oscillating field, see below.

The approach based on a $Z$ rotation is compared in Fig. 2 with the one based on adding to $H_{0}$ the counterdiabatic term $H_{c d}^{(1)}$ with the Landau-Zener scheme for $H_{0}$ (i.e., a constant $X_{0}$ and a linear in time $Z_{0}$ ). The process time is chosen to be short so that adiabaticity and population inversion fail for the Hamiltonian $H_{0}$ alone while these two approaches lead to perfect population inversion. In Fig. 2, it can be seen that their $Z$ components are similar, but the $X$ components have a rather different structure. A possible advantage of the superadiabatic + counterdiabatic approach using $H_{0}+H_{c d}^{(1)}$ is the smaller value of the $X$ maximum, which reduces amplitude noise and the field intensity.

Two-level atoms. - In quantum optics, Eq. (1), with $X_{0}=\hbar \Omega_{R} / 2, Y_{0}=0$, and $Z_{0}=-\hbar \Delta / 2$, represents a rotating frame IP Hamiltonian for a two-level atom in an oscillating field with angular frequency $\omega(t)=\omega_{0}-\Delta(t)$, where $\omega_{0}$ is the (angular) transition frequency, $\Omega_{R}$ the (on-resonance) Rabi frequency and $\Delta$ the detuning, after having applied the electric dipole and rotating wave approximations (RWAs). For $K=K_{L}=-[\hbar \omega(t) / 2] \sigma_{z}$ and $U=U_{L}=\exp \left[-(i / \hbar) \int_{0}^{t} K_{L}\left(t^{\prime}\right) d t^{\prime}\right]$ the corresponding $S$ (RWA) Hamiltonian is

$$
K_{L}+U_{L} H_{0} U_{L}^{\dagger}=\frac{\hbar}{2}\left(\begin{array}{cc}
-\omega_{0} & \Omega_{R} e^{i \theta} \\
\Omega_{R} e^{-i \theta} & \omega_{0}
\end{array}\right),
$$

where $\theta(t)=\int_{0}^{t} \omega\left(t^{\prime}\right) d t^{\prime}$. We can read from it the timedependent intensity, proportional to $\Omega_{R}^{2}$, the frequency of
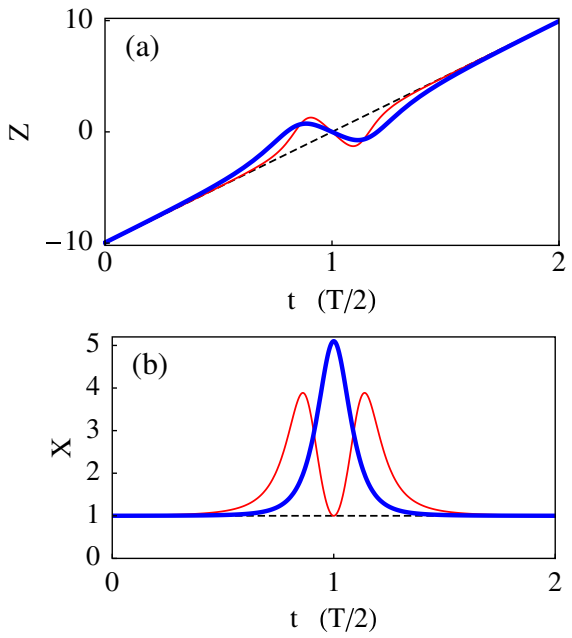

FIG. 2 (color online). $Z$ and $X$ Hamiltonian components for: $H_{0}$ (dashed lines); $z$-axis rotation Hamiltonian (4) (thick blue solid lines); superadiabatic + counterdiabatic method Hamiltonian $H_{0}+H_{c d}^{(1)}$ (thin red solid lines). $Z_{0}(t)=-\alpha(t-T / 2)$, and $\alpha=-10, T=20 /|\alpha|$, in units $\hbar=1, X_{0}=1$. 
the field that has to be applied, $\dot{\theta} /(2 \pi)$, and the atomic transition frequency $\omega_{0} /(2 \pi)$. If we start instead with $H_{0}+H_{c d}^{(0)}$ in the IP DE and apply the same transformation as before, we get the SP Hamiltonian

$$
\begin{aligned}
K_{L}+ & U_{L}\left(H_{0}+H_{c d}^{(0)}\right) U_{L}^{\dagger} \\
& =\frac{\hbar}{2}\left(\begin{array}{cc}
-\omega_{0} & \left(\Omega_{R}-i \dot{\Theta}_{0}\right) e^{i \theta} \\
\left(\Omega_{R}+i \dot{\Theta}_{0}\right) e^{-i \theta} & \omega_{0}
\end{array}\right),
\end{aligned}
$$

which requires in principle two fields dephased by $\pi / 2$ sharing a common time-dependent frequency but with different time-dependent intensities [5]. A $Z$ rotation may also be applied but realizing the result is now complicated due to the time dependence of the diagonal components, this would imply time-dependent level-shift engineering with an additional laser. It is thus advisable to find alternative, simpler realizations of the shortcuts.

$H_{0}+H_{c d}^{(0)}$ is not the only Hamiltonian that drives the populations along the ones of the adiabatic approximation for $H_{0}$. There is a whole family of them using different phases for the adiabatic base states, the simplest one being $H_{c d}^{(0)}$ itself $[16,18]$. Note that $A_{0}^{\dagger}\left(H_{c d}^{(0)}-K_{0}\right) A_{0}=0$ so the state does not move at all in the corresponding IP, whereas in the DE driven by $H_{c d}^{(0)}$ the populations will follow the ones for the adiabatic dynamics of $H_{0}$. By contrast, $H_{c d}^{(1)}$ alone is not enough to take the system along the superadiabatic path defined by $H_{1}$. We could still get rid of $H_{0}$ and use as a shortcut to superadiabaticity $H_{c d}^{(01)}:==$ $H_{c d}^{(0)}+H_{c d}^{(1)}$, but it combines the three Cartesian components.

Let us now take $H_{c d}^{(0)}=K_{0}$ as the reference IP Hamiltonian and try to implement it with different physical fields as in Fig. 1(b). Applying $U_{L}$ we get the SP Hamiltonian

$$
H_{S}=K_{L}+U_{L} K_{0} U_{L}^{\dagger}=\frac{\hbar}{2}\left(\begin{array}{cc}
-\omega(t) & -i \dot{\Theta}_{0} e^{i \theta} \\
i \dot{\Theta}_{0} e^{-i \theta} & \omega(t)
\end{array}\right),
$$

which is indeed problematic to realize because the atomic transition frequency should be time dependent. In other words, a simple IP Hamiltonian does not necessarily imply a simple experiment. To remedy this, keeping the same simple IP DE, we may use instead $U^{\prime}=e^{-(i / \hbar)} \int_{0}^{t} K^{\prime}\left(t^{\prime}\right) d t^{\prime}$, with $K^{\prime}=-(\hbar / 2) \omega_{0} \sigma_{z}$. This choice implies now a simple resonant interaction with constant frequency $\omega_{0}$ and $S^{\prime}$ Hamiltonian

$$
H_{S^{\prime}}=K^{\prime}+U^{\prime} K_{0} U^{\prime \dagger}=\frac{\hbar}{2}\left(\begin{array}{cc}
-\omega_{0} & -i \dot{\Theta}_{0} e^{i \omega_{0} t} \\
i \dot{\Theta}_{0} e^{-i \omega_{0} t} & \omega_{0}
\end{array}\right) .
$$

Other single laser implementations may also be developed by starting instead with $H_{0}+H_{c d}^{(1)}$. The term $H_{c d}^{(1)}$ modifies the detuning and Rabi frequency so that the transformation $U_{L}$ would lead to a SP Hamiltonian with the same structure as Eq. (5), but with modified laser and Rabi frequencies.
An alternative to the superadiabatic iterations is the "invariants-based inverse engineering approach" [18].

Trap expansions. - A further example is a fast harmonic trap expansion, or compression, which is receiving much attention because of fundamental and practical implications [1,19-26]. The reference Hamiltonian is $H_{h}=p^{2} /(2 m)+m \tilde{\omega}^{2} q^{2} / 2$, where $\tilde{\omega}=\tilde{\omega}(t)$ is the time-dependent angular frequency, $m$ the particle mass, and $q$ and $p$ are position and momentum operators. The corresponding counterdiabatic term to avoid excitations is $H_{c d}^{(0)}=-(p q+q p) \dot{\tilde{\omega}} /(4 \tilde{\omega})$, whose direct laboratory implementation is problematic and was left as an open question [6]. This difficulty is overcome by the transformation $U_{q}=\exp \left(i \frac{m \dot{\tilde{\omega}}}{4 \hbar \tilde{\omega}} q^{2}\right)$, which eliminates the cross terms; it produces from $H_{S}=H_{h}+H_{c d}^{(0)}$ the IP Hamiltonian $H_{I}=U_{q}^{\dagger}\left(H_{S}-i \hbar \dot{U}_{q} U_{q}^{\dagger}\right) U_{q}=p^{2} /(2 m)+$ $m \tilde{\omega}^{\prime 2} q^{2} / 2$, where $\quad \tilde{\omega}^{\prime}=\left[\tilde{\omega}^{2}-\frac{3 \tilde{\omega}^{2}}{4 \tilde{\omega}^{2}}+\frac{\tilde{\omega}}{2 \tilde{\omega}}\right]^{1 / 2}$. This Hamiltonian can actually be realized directly [20,21] and considered in a different physical setting as an ordinary harmonic oscillator with modified frequency. (This is different from applying the transformation just to solve formally the dynamics with $H_{S}$, see e.g., [27].) To satisfy the scheme of Fig. 1(b) we may apply $U^{\prime}=\mathbb{1}$ and regard $H_{I}$ as a Schrödinger Hamiltonian $S^{\prime}$, namely $H_{I}=H_{S^{\prime}}$. It indeed provides a shortcut with the following properties: Starting with a common state at time $t=0$, the spatial densities driven by $H_{h}+H_{c d}^{(0)}$ and $H_{S^{\prime}}$ are identical. In fact, by imposing $\dot{\tilde{\omega}}\left(t_{f}\right)=\ddot{\tilde{\omega}}\left(t_{f}\right)=0$ the final state is also equal for both dynamics, even in phase, and the final vibrational state populations coincide with those of a slow adiabatic process.

Transport.-The final example is harmonic transport of a particle [28-31]. A Hamiltonian $H_{0}=p^{2} /(2 m)+$ $\left[q-q_{0}(t)\right]^{2} m \tilde{\omega}_{0}^{2} / 2$, with $\tilde{\omega}_{0}$ constant, will generally induce excitations when moving the trap center $q_{0}(t)$. They are formally avoided by adding the counterdiabatic term $H_{c d}^{(0)}=$ $p \dot{q}_{0}$ in $H_{S}=H_{0}+H_{c d}^{(0)}$, which is, however, not realizable [30]. The transformation $U=e^{-i m \dot{q}_{0} q / \hbar}$ produces, after adding a time-dependent constant term, the feasible Hamiltonian $H_{I}=H_{S^{\prime}}=p^{2} /(2 m)+\left[q-q_{0}^{\prime}(t)\right]^{2} m \tilde{\omega}_{0}^{2} / 2$, where $q_{0}^{\prime}=q_{0}+\ddot{q}_{0} / \tilde{\omega}_{0}^{2}$. In this and previous examples a Lie algebra of operators and the Baker-Haussdorff identity facilitate the elimination of physically undesirable terms.

Discussion.-We have first proposed schemes for which an interaction picture dynamical equation represents different physical processes and interactions. These schemes have been later combined and exemplified to produce better, realizable shortcuts to adiabaticity for population inversion protocols, for trap expansions and compressions, and for harmonic transport. Similar manipulations may be applied as well to facilitate or improve shortcuts to adiabaticity for other operations and may be extended to three-level applications [32,33]. In fact the idea of designing the pictures to generate alternative, easier 
to handle physics, is applicable to a plethora of quantum systems, in particular, in the realms of quantum simulations, quantum control, or quantum information, where developing techniques to drive the systems for specific goals is a central objective.

We are grateful to M. V. Berry, M. Demirplak, D. GuéryOdelin, and O. Morsch for discussions. We acknowledge funding by Projects No. GIU07/40, No. FIS2009-12773C02-01, No. 61176118, and No. 12QH1400800, Juan de la Cierva Program, and the UPV/EHU under program UFI 11/55. S.I. and E.T. acknowledge financial support from the Basque Government (Grants No. BFI09.39 and No. BFI08.151).

[1] X. Chen, A. Ruschhaupt, S. Schmidt, A. del Campo, D. Guéry-Odelin, and J.G. Muga, Phys. Rev. Lett. 104, 063002 (2010).

[2] X. Sun, Hsi-Chun Liu, and A. Yariv, Opt. Lett. 34, 280 (2009).

[3] M. Deschamps, G. Kervern, D. Massiot, G. Pintacuda, L. Emsley, and P. J. Grandinetti, J. Chem. Phys. 129, 204110 (2008).

[4] B. T. Torosov, S. Guérin, and N. V. Vitanov, Phys. Rev. Lett. 106, 233001 (2011).

[5] X. Chen, I. Lizuain, A. Ruschhaupt, D. Guéry-Odelin, and J. G. Muga, Phys. Rev. Lett. 105, 123003 (2010).

[6] J. G. Muga, X. Chen, S. Ibáñez, I. Lizuain, and A. Ruschhaupt, J. Phys. B 43, 085509 (2010).

[7] L. M. Garrido, J. Math. Phys. (N.Y.) 5, 355 (1964).

[8] M. V. Berry, Proc. R. Soc. A 414, 31 (1987).

[9] M. Demirplak and S. A. Rice, J. Chem. Phys. 129, 154111 (2008).

[10] A. Zenesini, H. Lignier, G. Tayebirad, J. Radogostowicz, D. Ciampini, R. Mannella, S. Wimberger, O. Morsch, and E. Arimondo, Phys. Rev. Lett. 103, 090403 (2009).

[11] M. G. Bason, M. Viteau, N. Malossi, P. Huillery, E. Arimondo, D. Ciampini, R. Fazio, V. Giovannetti, R. Mannella, and O. Morsch, Nature Phys. 8, 147 (2012).
[12] L. Allen and J.H. Eberly, Optical Resonance and TwoLevel Atoms (Dover, New York, 1987).

[13] A. Messiah, Quantum Mechanics (Dover, New York, 1999).

[14] M. Demirplak and S. A. Rice, J. Phys. Chem. A 107, 9937 (2003).

[15] M. Demirplak and S. A. Rice, J. Phys. Chem. B 109, 6838 (2005).

[16] M. V. Berry, J. Phys. A 42, 365303 (2009).

[17] M. V. Berry, Proc. R. Soc. A 429, 61 (1990).

[18] X. Chen, E. Torrontegui, and J. G. Muga, Phys. Rev. A 83, 062116 (2011).

[19] P. Salamon, K. H. Hoffmann, Y. Rezek, and R. Kosloff, Phys. Chem. Chem. Phys. 11, 1027 (2009).

[20] J. F. Schaff, X. L. Song, P. Vignolo, and G. Labeyrie, Phys. Rev. A 82, 033430 (2010); 83, 059911(E) (2011).

[21] J. F. Schaff, X. L. Song, P. Capuzzi, P. Vignolo, and G. Labeyrie, Europhys. Lett. 93, 23001 (2011).

[22] S. Masuda and K. Nakamura, Proc. R. Soc. A 466, 1135 (2010).

[23] X. Chen and J.G. Muga, Phys. Rev. A 82, 053403 (2010).

[24] D. Stefanatos, J. Ruths, and J.-S. Li, Phys. Rev. A 82, 063422 (2010).

[25] A. del Campo, Phys. Rev. A 84, 031606(R) (2011).

[26] K. H. Hoffmann, P. Salamon, Y. Rezek, and R. Kosloff, Europhys. Lett. 96, 60015 (2011).

[27] A. Mostafazadeh, J. Phys. A 31, 6495 (1998).

[28] A. Couvert, T. Kawalec, G. Reinaudi, and D. GuéryOdelin, Europhys. Lett. 83, 13001 (2008).

[29] M. Murphy, L. Jiang, N. Khaneja, and T. Calarco, Phys. Rev. A 79, 020301(R) (2009).

[30] E. Torrontegui, S. Ibáñez, X. Chen, A. Ruschhaupt, D. Guéry-Odelin, and J. G. Muga, Phys. Rev. A 83, 013415 (2011).

[31] R. Bowler, J. Gaebler, Y. Lin, T. R. Tan, D. Hanneke, J. D. Jost, J. P. Home, D. Leibfried, and D. J. Wineland, Phys. Rev. Lett. 109, 080502 (2012).

[32] A. Kuhn, M. Hennrich, and G. Rempe, Phys. Rev. Lett. 89, 067901 (2002).

[33] M. Hijlkema, B. Weber, H.P. Specht, S. C. Webster, A. Kuhn, and G. Rempe, Nature Phys. 3, 253 (2007). 